\title{
NORMATIVE VALUES FOR DRIVING SIMULATION PARAMETERS: A PILOT STUDY
}

\author{
Abiodun Emmanuel Akinwuntan, Rebecca Tank, \\ Lori Vaughn, Alexis Wilburn, Seth Easton \\ Medical College of Georgia \\ Augusta, Georgia 30912 \\ E-mail: aakinwuntan@mcg.edu
}

\begin{abstract}
Summary: In this pilot study, data obtained from a population of healthy younger (18-24 yrs), middle aged (25-64 yrs) and older drivers (65+ yrs) were used to establish normative values of parameters commonly documented during simulated driving. The older drivers' performances in most of the driving skills assessed were significantly worse than those of the other 2 classes of drivers. In line with previous studies, our data showed deterioration of driving skills with increasing age. The test-retest reliability of the driving simulation parameters were moderate to very high.
\end{abstract}

\section{INTRODUCTION}

Studies have shown that driving simulators are useful in the evaluation of on-road driving abilities of older individuals (Freund et al., 2002; Lee, Lee, 2005) and after neurological impairments (Kotterba et al., 2003; Devos et al., 2007). Some driving skills commonly assessed during simulator-based driving evaluations include lane tracking, gap estimation (time and range to collision), accident avoidance, compliance with speed limits and reaction time. We undertook this study to establish normative values of these driving skills during navigation of a 9.5 mile scenario and another 1.5 mile scenario that we routinely use as part of the evaluation of on-road driving ability in our laboratory. Availability of normative values, we believe will further enhance our appreciation of the extent of deficits in the driving skills of our clients and guide the decision on the best way forward. We also hope that it is the first step towards developing a standardized scenario with traffic events that will generate widely acceptable and used normative values.

\section{METHODS}

Participants in the pilot study were healthy individuals who had valid driver licenses, had at least 2 years driving experience and drove at least 10 miles weekly. To be considered healthy, participants had to be devoid of any neurological problem and demonstrate sufficient physical, mental and visual fitness to drive. To ensure that participants met these and other study criteria, each participant and the study investigator completed a comprehensive questionnaire that had 3 parts. The first part of the questionnaire was on demographic details such as class of license, driving experience, average distance driven annually and daily, level of education and medical history. The second part of the questionnaire, in which the physical ability, mental state, cognitive-perceptual skills and visual acuity were evaluated, was completed by the study investigator. Functional strength and active range of motion of both upper and lower extremities, functional balance, coordination and independence in performing activities of daily living using 
the Barthel Index were used as measures of physical ability. The Mini Mental State Examination was used to establish mental state. Cognitive and perceptual skills were evaluated using ReyOsterrieth Complex Figure and Trail Making Tests A \& B. Participants were considered healthy only when they met or were better than established normative values for their respective age categories in the assessments. Visual requirements were in accordance with the state of Georgia's driving laws, which stipulates binocular visual acuity of at least 20/60. The third part of the questionnaire contained 3 copies of the simulator sickness questionnaire published by Kennedy et al., 1993. Particpants were instructed to complete the first copy by indicating the extent to which they had ever experienced any of the symptoms of simulation sickness listed in the questionnaire. The second copy was to indicate how particpants currently felt and the third copy was to be completed after completion of the driving simulation.

Only those that met all study criteria and were considered not likely to become simulator sick were further assessed in a STISIM Model 300 driving simulator incorporated into a full-sized Plymouth Acclaim, 1991 sedan car. While in the simulator, participants were instructed in a standardized manner to use all operational parts (steering wheel, gas and brake pedals, seat belt and turn signals) of the car as in real life to navigate presented simulated driving scenarios. To get familiarized with driving simulation, participants first drove through a simulated 2 mile scenario containing simple traffic events which was projected with 50 degrees field of view on a 9 square feet screen mounted on a wall 2 inches in front of the stationary car (figure 1). After verbal declaration of familiarization, participants were presented a 9.5 mile long scenario. The scenario contained simulation of a regular 10.30 am work day traffic stream on a popular route around Augusta, GA (figure 2) which was previously recorded on video. The route predominantly comprised of 4-lane (2 lanes in each direction) undivided urban/city roads with 12 signal controlled intersections and an interstate highway with 2 entry/exit single lane ramps. Speed limits along the route ranged from 30 to $70 \mathrm{mph}$.

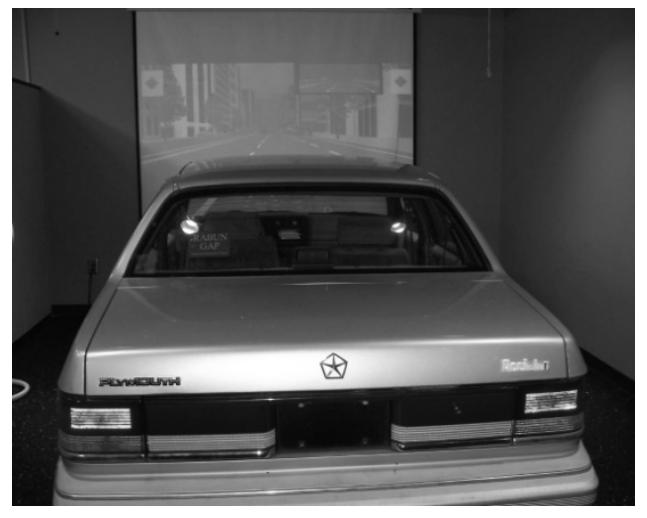

Fig. 1 Driving Simulator Set-up

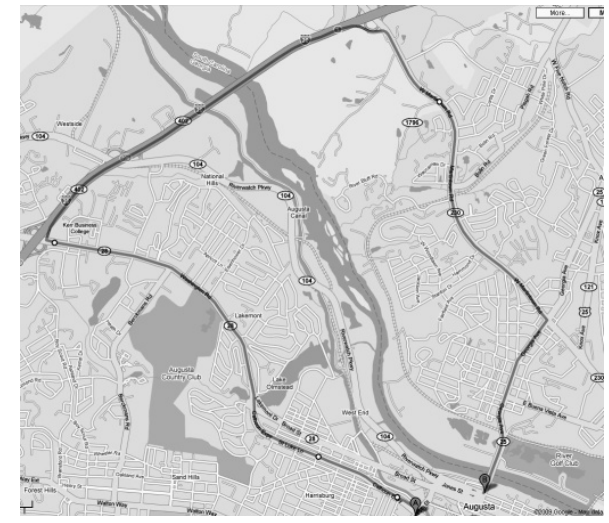

Fig. 2. Aerial View of 9.5 mile Route

For further route details, contact first author. Data including number of road edge excursions and centerline crossings, average time to collision (TTC) with lead vehicles, number of collisions, number of speed excesses and time to complete the 9.5 mile scenario (run time) were automatically collected in the computer attached to the simulator during each drive.

Number of road edge excursions was the number of times any of the 2 right wheels of the driver made contact with any part of the outer right road side line. Number of center line crossings was 
the number of times any of the 2 left wheels of the driver made contact with any part of the center line closer to the driver on the undivided road or left road side line on the interstate highway and exit/entry ramps. Time to collision (TTC) data referred to the average, in seconds, of how close the driver's vehicle got to all other vehicles in the driver's path during the drive. Number of collisions was the number of times the driver collided with a lead vehicle or object. Number of speed excesses represented the number of times the specified speed limit was exceeded by at least $5 \mathrm{mph}$. Run time was the time spent in seconds from start to finish of the simulated 9.5 mile scenario.

After successfully completing the 9.5 mile scenario, participants were presented a third scenario that was 1.5 mile long and had only a 2-lane road (single lane in each direction) with $45 \mathrm{mph}$ speed limit signs posted by the right road side. In the scenario, 5 large 'STOP' signs appeared at specific but different times in the middle of the screen. Before driving through the scenario, participants were instructed to adhere to the speed limit and to react as fast as possible to the 'STOP' signs each time by bringing the car to a complete stop and resume normal driving afterwards. The scenario was used to assess simple reaction time. Four variables namely seeing

time, movement time, braking time and reaction time were derived from the data collected. Seeing time was calculated as the time from presentation of each sign to the beginning of a significant decrease in the throttle input counts. Movement time was the duration between the significant decrease in the throttle input counts and significant increase in brake input counts. Braking time was calculated as duration between the significant increase in brake input counts to when speed dropped to zero. Reaction time was calculated as the time from presentation of the 'STOP' stimuli to when the car was brought to a complete stop. For each variable, the average value from the 5 presentations of the sign was used in the data analysis.

To determine the test-retest reliability of the driving simulation data, 15 randomly chosen participants volunteered and returned between 60 and 90 days after their initial participation to drive the simulated scenarios again following exactly the same procedures described above.

\section{RESULTS}

A total of 153 individuals volunteered to particpate in the study over a period of 18.5 months. Two individuals scored below their age norms in the cognitive and perceptual tests and were not allowed to proceed in the study. Of the 151 that met all study criteria, data of 21 participants $(13.9 \%)$ were not included in the analyses to establish normative values due to simulation sickness related issues. Correlation statistics using descriptive variables of the remaining 130 participants showed that age was the single most correlated variable with the simulation parameters. Regression analyses also revealed age as the most important predictor of majority of the parameters. As such, we stratified our sample into 3 classes based on age groups commonly reported in literature: 18-24 years or younger drivers, 25-64 years or middle aged drivers, and $65+$ years or older drivers. The appropriateness of the 3 -level stratification was confirmed by the outcome of several comparisons to investigate if any other levels of stratification was better.

Study data revealed that only 5 participants ( 3 middle aged and 2 older drivers) each crossed the centerline one time, and all were very early in the scenario. There were 6 incidences of collisions, each by 3 younger and 3 middle aged drivers, with the barrier that was used to 
simulate entry ramp that linked the outer right lane of the 4-lane undivided road to the 4-lane interstate highway. None of the 130 participants collided with any lead vehicle. Normative values of the other variables in the 3 age classes are presented in tables $1 \mathrm{a}, 1 \mathrm{~b}$ and $1 \mathrm{c}$.

Table 1a. Normative values in younger drivers

\begin{tabular}{lllll}
\hline Variables & \multicolumn{5}{c}{ 18-24 years, $\mathrm{n}=29$} \\
\hline Age, yrs & Mean \pm SD & Med(Min-Max) & $90^{\text {th }}$ \%ile & Skew \\
\cline { 2 - 5 } Road edge excursions, $\mathrm{n}$ & $22.7 \pm 1.3$ & $23.0(19.0-24.0)$ & 24 & -1.0 \\
TTC, sec & $19.7 \pm 8.2$ & $18.0(5.0-37.0)$ & 35 & 0.4 \\
Speed excesses, $\mathrm{n}$ & $2.0 \pm 0.3$ & $2.1(1.2-2.6)$ & 2.3 & -1.1 \\
Run time, sec & $5.8 \pm 2.8$ & $5.0(2.0-13.0)$ & 9 & 0.9 \\
\hline
\end{tabular}

Table 1b. Normative values in middle aged drivers

\begin{tabular}{llllc}
\hline Variables & \multicolumn{4}{c}{ 25-64 year, $\mathrm{n}=73$} \\
\cline { 2 - 5 } Age, yrs & \multicolumn{1}{c}{ Mean \pm SD } & \multicolumn{1}{c}{ Med(Min-Max) } & $90^{\text {th }}$ \%ile & Skew \\
\cline { 2 - 5 } Road edge excursions, $\mathrm{n}$ & $39.5 \pm 10.0$ & $37.0(25.0-64.0)$ & 55 & 0.4 \\
TTC, sec & $20.7 \pm 8.1$ & $20.0(5.0-46.0)$ & 32 & 0.5 \\
Speed excesses, $\mathrm{n}$ & $2.1 \pm 0.3$ & $2.1(1.2-2.5)$ & 2.4 & -0.5 \\
Run time, sec & $5.2 \pm 2.7$ & $5.0(0.0-14.0)$ & 9 & 0.5 \\
\hline
\end{tabular}

Table 1c. Normative values in older drivers

\begin{tabular}{lllll}
\hline Variables & \multicolumn{4}{c}{ 65+ years, $\mathrm{n}=28$} \\
\hline Age, yrs & Mean \pm SD & Med(Min-Max $)$ & $90^{\text {th }}$ \%ile & Skew \\
\cline { 2 - 5 } Road edge excursions, $\mathrm{n}$ & $69.9 \pm 4.2$ & $69.0(65.0-79.0)$ & 77 & 0.8 \\
TTC, sec & $27.5 \pm 9.4$ & $27.0(11 .-48.0)$ & 41 & 0.3 \\
Speed excesses, $\mathrm{n}$ & $2.2 \pm 0.3$ & $2.19(1.81-3.13)$ & 2.5 & 1.5 \\
Run time, sec & $5.0 \pm 3.7$ & $4.0(0.0-15.0)$ & 11 & 1.1 \\
\hline
\end{tabular}

Normative values for each parameter was compared between groups and where there were significant differences, post hoc analysis was done to identify which 2 groups were different (table 1d).

Table 1d: Comparisons between normative values in the 3 classes of drivers

\begin{tabular}{lll}
\hline Variables & \multicolumn{1}{c}{1 vs 2 vs 3} & Post hoc \\
\hline Age, yrs & $\mathrm{K}=103.56, \mathrm{p}=<.0001$ & $1 * 2,2 * 3,1 * 3$ \\
Road edge excursions, $\mathrm{n}$ & $\mathrm{K}=13.35, \mathrm{p}=0.0013$ & $1 * 3,2 * 3$ \\
TTC, sec & $\mathrm{K}=8.59, \mathrm{p}=0.01$ & $1 * 3,2 * 3$ \\
Speed excesses, $\mathrm{n}$ & $\mathrm{K}=2.05, \mathrm{p}=0.36$ & \\
Run time, sec & $\mathrm{K}=19.58, \mathrm{p}=<.0001$ & $1 * 2,2 * 3,1 * 3$ \\
\hline
\end{tabular}

$\mathrm{K}=$ Kruskal Wallis

Older drivers (65+ yrs) had significantly more number of road edge excursions, longer times to collision with lead vehicles, and longer times to complete the 9.5 mile scenario when compared to the other 2 classes of drivers.

Normative values for the seeing time, movement time, braking time and reaction time to the 'STOP' signs presented in the 1.5 mile scenario are detailed in tables $2 \mathrm{a}, 2 \mathrm{~b}$ and $2 \mathrm{c}$ and are based 
on performances of 120 participants. Data of 10 of the 130 participants could not be used due to technical difficulties retrieving the data files.

Table 2a. Normative values of reaction time in younger drivers at average $\mathrm{speed}=\mathbf{4 5} \mathbf{~ m p h}$

\begin{tabular}{lllll}
\hline Variables & \multicolumn{5}{c}{$18-24$ years, $\mathrm{n}=27$} \\
\hline & Mean \pm SD & Med(Min-Max) & $90^{\text {th }} \%$ ile & Skew \\
\cline { 2 - 5 } Age, yrs & $22.7 \pm 1.3$ & $23.0(19.0-24.0)$ & 24 & -1.10 \\
Seeing time, sec & $0.45 \pm 0.08$ & $0.43(0.35-0.71)$ & 0.56 & 1.46 \\
Movement time, sec & $0.31 \pm 0.17$ & $0.29(0.13-0.90)$ & 0.52 & 2.04 \\
Braking time, sec & $3.20 \pm 0.42$ & $3.17(2.33-4.07)$ & 3.93 & 0.14 \\
Reaction time, sec & $3.97 \pm 0.53$ & $3.63(2.96-5.09)$ & 4.96 & 0.65 \\
\hline
\end{tabular}

Table $2 \mathrm{~b}$. Normative values of reaction time in middle aged drivers at average $\mathbf{s p e e d}=45 \mathrm{mph}$

\begin{tabular}{lllll}
\hline Variables & \multicolumn{4}{c}{$25-64$ years, $\mathrm{n}=65$} \\
\hline Age, yrs & Mean \pm SD & Med(Min-Max) & $90^{\text {th }}$ \%ile & Skew \\
\cline { 2 - 5 } Seeing time, sec & $39.1 \pm 1.0$ & $37.0(25.0-64.0)$ & 55 & 0.41 \\
Movement time, sec & $0.47 \pm 0.07$ & $0.45(0.33-0.68)$ & 0.56 & 0.93 \\
Braking time, sec & $3.21 \pm 0.12$ & $0.25(0.07-0.63)$ & 0.54 & 0.12 \\
Reaction time, sec & $3.94 \pm 0.45$ & $3.15(2.57-4.26)$ & 3.68 & 0.66 \\
\hline
\end{tabular}

Table 2c. Normative values of reaction time in older drivers at average $\mathrm{speed}=45 \mathrm{mph}$

\begin{tabular}{lllll}
\hline Variables & \multicolumn{5}{c}{$65+$ years, $\mathrm{n}=28$} \\
\hline & Mean \pm SD & Med(Min-Max) & $90^{\text {th }}$ \%ile & Skew \\
\cline { 2 - 5 } Age, yrs & $69.7 \pm 4.3$ & $68.5(65.0-79.0)$ & 77 & 0.81 \\
Seeing time, sec & $0.55 \pm 0.08$ & $0.55(0.38-0.68)$ & 0.66 & -0.31 \\
Movement time, sec & $0.26 \pm 0.13$ & $0.25(0.03-0.52)$ & 0.43 & 0.11 \\
Braking time, sec & $3.23 \pm 0.51$ & $3.07(2.64-4.68)$ & 4.14 & 1.70 \\
Reaction time, sec & $4.03 \pm 0.58$ & $3.65(3.41-5.62)$ & 5.33 & 1.70 \\
\hline
\end{tabular}

On average, older drivers took the longest time ( 0.55 seconds) to take the foot off the gas pedal in reaction to 'STOP' signs when compared to younger $(0.45$ seconds $)$ and middle aged drivers (0.47 seconds).

Table 2d. Comparisons between normative values reaction time in the 3 classes of drivers

\begin{tabular}{lll}
\hline Variables & 1 vs 2 vs 3 & Post hoc \\
\hline Age, yrs & $\mathrm{K}=97.34, \mathrm{p}=<.0001$ & $1 * 2,2^{*} 3,1 * 3$ \\
Seeing time, sec & $\mathrm{K}=25.38, \mathrm{p}=<.0001$ & $1 * 3,2 * 3$ \\
Movement time, sec & $\mathrm{K}=1.18, \mathrm{p}=0.56$ & \\
Braking time, sec & $\mathrm{K}=0.62, \mathrm{p}=0.73$ & \\
Reaction time, sec & $\mathrm{K}=0.05, \mathrm{p}=0.97$ & \\
\hline
\end{tabular}

$\mathrm{K}=$ Kruskal Wallis

Outcomes of the intraclass correlation coefficient analyses performed using the first and second trials data of the 15 participants that were assessed twice to establish the test-retest reliability of the simulation data are presented in table 3. With the exception of road edge excursions, all other simulation parameters were moderate to very highly reliable. 
Table 3. Intraclass Correlation Coeeficients (ICC) and 95\% Confidence Interval (CI) of the simulation parameters

\begin{tabular}{lcccc} 
Variables & T1:Mean \pm SD & T2: Mean \pm SD & ICC & $95 \%$ CI \\
\hline 10.5 Mile Scenario & & & & \\
Road edge excursions, $\mathrm{n}$ & $16.8 \pm 4.9$ & $16.2 \pm 9.7$ & 0.20 & $-1.67-0.74$ \\
TTC, sec & $2.0 \pm 0.3$ & $2.1 \pm 0.5$ & 0.55 & $-0.38-0.85$ \\
Speed excesses, $\mathrm{n}$ & $4.5 \pm 1.4$ & $5.3 \pm 2.6$ & 0.72 & $0.21-0.90$ \\
Run time, sec & $800.9 \pm 93.5$ & $773.9 \pm 124.8$ & 0.70 & $0.13-0.90$ \\
Simple Reaction Time & & & & \\
Seeing time, sec & $0.49 \pm 0.05$ & $0.51 \pm 0.07$ & 0.69 & $0.13-0.89$ \\
Movement time, sec & $0.32 \pm 0.11$ & $0.35 \pm 0.12$ & 0.71 & $0.16-0.90$ \\
Braking time, sec & $3.25 \pm 0.31$ & $3.30 \pm 0.33$ & 0.88 & $0.65-0.96$ \\
Reaction time, sec & $4.02 \pm 0.38$ & $4.12 \pm 0.33$ & 0.88 & $0.63-0.96$ \\
\hline
\end{tabular}

$\mathrm{T} 1=$ Trial 1 (first trial), $\mathrm{T} 2=$ Trial 2 (second trial)

\section{DISCUSSION}

Participants included in this pilot study were healthy persons with at least 2 years driving experience and actively drove at least 10 miles a week. It can thus be expected that they are safe enough drivers to avoid collision with lead vehicles. The 6 particpants that collided with the barrier claimed after completing the scenario that they got confused when they approached the only major unnatural section of the road and did not know what to do. That the 5 particpants who each had an episode of centerline crossing early on in the 9.5 mile scenario self corrected and never did it again again the rest of the drive led us to conclude that they were probably still getting fully familiarized with driving simulation in the early stages of the drive. Older drivers when compared to the other 2 classes of drivers, had more difficulty keeping the car on the road without crossing the side line. However, the older drivers exercised greatest caution when approaching a lead vehicle. These findings suggest a tactic of keeping safe distance from other vehicles on the road, a known compensaory method commonly adopted by older drivers and also exhibited in the study by Yan et al., 2007. In spite of participants in the 3 classes exceeding the posted speed limit similar number of times, the younger drivers posted fastest time to complete 9.5 mile scenario. This could mean the younger driver were willing to take more risks during the simulated drive as they are known to do during real life driving by consistently staying close to the speed limit.

In the simple reaction time scenario, older drivers took the longest time to raise the foot off the gas pedal from the times the 'STOP' signs were presented (seeing time). Since the signs were so big that they covered almost two-thirds of the screen when presented, this finding could not be attributed to participants' visual acuity. Rather we believe that the finding is due to slowness in speed of mental processing as a result of aging. Fildes et al., 2007; Warshawsky-Livne and Shinar, 2002 also found that driving-related visual perception deteriorates with increasing age. It could also be due to slowness in activating and executing the motor activity of raising the foot from the pedal (foot reaction time). In the review study by Anstey et al, 2005, increased foot reaction time positively assicated with increasing age. It is also possible that the finding is as a result of both reasons combined. Despite the longest seeing time, overall reaction time to the 'STOP' signs presented during driving at about $45 \mathrm{mph}$ by the older drivers was similar to the reaction times by the younger and middle aged drivers. Older drivers probably compensated for their decline in mental and physical functions by not over speeding as was the case in the other 2 
classes of drivers. Speed adaptation is also a compensatory mechanism commonly used by older drivers during driving.

Moderate to high reliability of most of the simulation data suggest that the simulation data is reliable.

The number of participants used to establish the normative values, the reliability of the simulation data and the huge differences in number of younger and older participants, when compared to middle aged drivers represent some of the limitations of this pilot study. None inclusion of completely unpredictable events to assess complex reaction time, divided attention and excutive function in the 9.5 mile scenario, especially at intersections, is also a limitation. We plan to address these limitations in future studies.

\section{ACKNOWLEDGMENT}

The authors acknowledge the Sponsored Program Administration of the Medical College of Georgia, Augusta GA for funding this study.

\section{REFERENCES}

Anstey, K.J., Wood, J., Lord, S. \& Walker, J.G. (2005). Cognitive, sensory, and physical factors enabling driving safety in older adults. Clinical Psychology Review, 25(1), 45-65.

Devos, H., Vandenberghe, W., Nieuwboer, A., Tant, M., Baten, G., De Weerdt, W. (2007). Predictors of fitness to drive in people with Parkinson disease. Neurology, 69 (14),14341441.

Fildes, B., Charlton, J., Muir, C., \& Kopple, S. ( 2007). Driving responses of older and younger drivers in a driving simulator. Abstract Only. Annual Proceedings Association for the Advancement for Automotive Medicine 51, 559-572.

Freund, B., Gravenstein, S., Ferris, R. \& Shaheen, E. ( 2002). Evaluating driving performance of cognitively impaired and healthy older adults: a pilot study comparing on-road testing and driving simulation. Journal of the American Geriatrics Society, 50(7), 1309-1310.

Kennedy, R.S., Lane, N.E., Berbaum, K.S.(1993). Simulator Sickness Questionnaire: An Enhanced Method for Quantifying Simulator Sickness. International Journal of Aviation Psychology, 3(3), 203-220.

Kotterba, S., Orth, M., Eren, E., Fangerau, T., Sindern, E. (2003). Assessment of driving performance in patients with relapsing-remitting multiple sclerosis by a driving simulator. European Journal of Neurology, 50(3), 160-164.

Lee, H.C. \& Lee, A.H.. (2005). Identifying older drivers at risk of traffic violations by using a driving simulator: a 3-year longitudinal study. American Journal of Occupational Therapy, 59(1), 97-100.

Yan, X., Radwan, E., \& Guo, D. (2007). Effects of major-road vehicle speed and driver age and gender on left-turn gap acceptance. Accident Analysis and Prevention, 39(4), 843-852. 
PROCEEDINGS of the Fifth International Driving Symposium on Human Factors in Driver Assessment, Training and Vehicle Design

Warshawsky-Livne, L. \& Shinar, D., 2002. Effects of uncertainty, transmission type, driver age and gender on brake reaction and movement time. Journal of Safety Research, 33(1), $117-$ 128. 